\title{
CARACTERIZACIÓN DE LOS DESENLACES MATERNOS DE LA HEMORRAGIA POSPARTO PRIMARIA EN UN HOSPITAL DE MONTERÍA, COLOMBIA, 2016
}

\author{
CHARACTERIZATION OF MATERNAL OUTCOMES OF PRIMARY POSTPARTUM \\ HEMORRHAGE IN A HOSPITAL IN MONTERIA, COLOMBIA, 2016.
}

\author{
Javier Bula Romero¹, María del Pilar Guzmán Arteaga², Álvaro Sánchez Caraballo³
}

Recibido para publicación: Octubre 6 de 2017 - Aprobado para publicación: Diciembre 12 de 2017

\begin{abstract}
RESUMEN
Objetivo: Describir las características demográficas, las intervenciones realizadas y desenlaces maternos en las mujeres que presentaron hemorragia en las primeras 24 horas posparto o poscesárea en un hospital de mediana complejidad de Montería del departamento de Córdoba. Metodología: Estudio descriptivo retrospectivo, de tipo exploratorio basado en el análisis de registros clínicos. Resultados: La edad de las pacientes osciló entre 13 - 42 años, la mayoría eran multíparas, más del 50\% de las pacientes vivían en unión libre y residía en el área urbana. El 75\% de los casos identificados fueron remitidos de los primeros Niveles de Atención en salud del departamento de Córdoba. La retención de tejidos y los traumatismos en el canal vaginal fueron las principales causas de hemorragia postparto, el $50 \%$ de los casos requirieron transfusión sanguínea con glóbulos rojos O Rh -, el $17 \%$ requirió de ingreso a una $\mathrm{UCl}$ y el $33 \%$ de los casos restantes necesitaron manejo quirúrgico, dos pacientes requirieron de histerectomía subtotal, ambas primigestantes adolescentes. Conclusiones: Las causas más frecuente de Hemorragia Posparto en los partos vaginales están relacionadas con traumatismos en el canal vaginal y con la retención de tejido placentario y restos de membranas ovulares.
\end{abstract}

Palabras clave: Hemorragia, posparto, Mortalidad, Materna.

\begin{abstract}
Objective: To describe the demographic characteristics and interventions performed as well as the maternal outcomes in women who presented hemorrhage in the first 24 hours postpartum or post-cesarean in a medium-complexity hospital in Monteria, Colombia, South America. Methodology: This was a retrospective, descriptive, and exploratory study based on the analysis of clinical records. Results: The patients' ages ranged between 13 and 42 years. The majority were multiparous; more than $50 \%$ of the patients lived in a consensual union and resided in the urban area. $75 \%$ of the cases identified were sent from the first levels of health care in the department of Córdoba. The issue retention and traumatisms in the vaginal canal were the central causes of postpartum hemorrhage. $50 \%$ of the cases required blood transfusion with red blood cells or Rh. $17 \%$ required admission to an ICU and $33 \%$ of the remaining cases required surgical management. Two patients required subtotal hysterectomy, and both were first-pregnancy adolescents. Conclusions: The most frequent causes of postpartum hemorrhage in vaginal deliveries are related to traumatisms in the vaginal canal and to the retention of placental tissue and remains of ovular membranes.
\end{abstract}

Keywords: Postpartum, hemorrhage, maternal, mortality.

\footnotetext{
${ }^{1}$ Enfermero M.Sc \& PhD (c) en Enfermería. Profesor Facultad Ciencias de la Salud, Universidad de Córdoba, Montería, Colombia. Correspondencia javierbula@correo.unicordoba.edu.co

${ }^{2}$ Enfermera M.Sc en Enfermería. Profesora Facultad Ciencias de la Salud, Universidad de Córdoba, Montería, Colombia.

${ }^{3}$ Enfermero M.Sc \& PhD en Salud Pública. Profesor Facultad Ciencias de la Salud, Universidad de Córdoba, Montería, Colombia.
} 


\section{INTRODUCCIÓN}

La Hemorragia posparto (HPP) es la causa más frecuente de mortalidad y morbilidad materna en el mundo; en los países desarrollados causa el $8 \%$ de las muertes, mientras que en los países en vía de desarrollo es responsable del $19.7 \%$ de las muertes maternas $(1,2,3)$. En Latinoamérica y el Caribe, la hemorragia posparto es responsable del $13.3 \%$ de las muertes y en Colombia, la tendencia se mantiene, siendo responsable del $23 \%$ de las muertes maternas (4). La prevención y el manejo de la hemorragia posparto, es en consecuencia un pilar fundamental para disminuir la mortalidad materna $(5,6,7,8,9)$.

Existen varias clasificaciones de hemorragia posparto de acuerdo a la cantidad de pérdida estimada: Es una hemorragia Posparto menor, si la perdida es de 500 a 1000 cc y mayor, cuando supera $1000 \mathrm{cc}$. De acuerdo al momento de presentación, puede ser: primaria; cuando se presenta en las primeras 24 horas, y secundaria después de las 24 horas o hasta las 6 semanas posparto (10). Otra de las clasificaciones se basa en el grado de choque y la magnitud de la perdida sanguínea, esta puede ser: compensada (500 cc a $1000 \mathrm{cc}$ ), leve (1000 a $1500 \mathrm{cc}$ ) moderado (1500 a 2000 cc) y severa más de 2000 cc) $(11,12,13,14)$.

El objetivo de este estudio fue describir las características demográficas, las intervenciones realizadas y desenlaces maternos en las mujeres que presentaron hemorragia en las primeras 24 horas posparto o poscesárea en un hospital de mediana complejidad de la ciudad de Montería del departamento de Córdoba.

\section{MATERIALES Y MÉTODOS}

Estudio descriptivo retrospectivo, de tipo exploratorio basado en el análisis de registros clínicos. Se realizó en el Hospital san Jerónimo de Montería Empresa Social del Estado, de III nivel de complejidad, que atiende pacientes del sistema de seguridad social de salud en el departamento de Córdoba entre el 1 enero y el 31 de diciembre de 2016.

Se incluyeron todas las mujeres que presentaron hemorragia las primeras 24 horas posparto o post cesárea o la activación de la respuesta organizada a la emergencia obstétrica por hemorragia post parto independientemente de la vía del parto. Se excluyeron las mujeres con hemorragia obstétrica debida a causas diferentes al parto o la cesárea.

Se realizó un registro de todas las pacientes atendidas por parto o cesárea que presentaron hemorragia posparto primaria, definida como un sangrado que requiera cualquier intervención adicional al manejo activo del tercer periodo del parto en las primeras 24 horas.

\section{Fase retrospectiva del estudio}

Para dar respuesta a la pregunta de investigación y llevar a cabo el cumplimiento de los objetivos del estudio, se solicitó inicialmente el permiso institucional para el desarrollo de la investigación.

La información de los casos de Hemorragia posparto primaria se obtuvo a partir de la revisión de las Historias clínicas y la base de datos de seguimiento a los casos de morbilidad materna extrema en la institución y las fichas de Notificación de Morbilidad Materna Extrema $M M E(15)$. Los datos recolectados fueron transcritos fielmente en una base de datos diseñada en Microsoft Excel versión 3.5 en la cual se condensó la información, previa verificación de los registros con el fin de identificar duplicidad de los datos o errores en la transcripción de los mismos. Por último la información no fue alterada ni sustituida en ningún momento del proceso, garantizando la veracidad de la información para el desarrollo del proyecto investigativo. 


\section{RESULTADOS}

Durante el periodo de recolección de datos se presentó un total de 2.594 nacimientos, 602 partos vaginales y 1992 cesáreas para una proporción del $76.8 \%$. Según la vía del parto, se reportaron 20 casos de HPP en partos vaginales (83\%) y cuatro casos de HPP en partos por vía abdominal o cesárea (17\%). La edad de las pacientes osciló entre 13 - 42 años, la mayoría eran multíparas, el $50 \%$ de las pacientes vivía en unión libre, y la mayor proporción de ellas residía en el área urbana, 16/24 (66\%). El 75\% de los casos de hemorragia posparto primaria fueron remitidos de los primeros Niveles de atención en salud del departamento de Córdoba. Como se puede obsertar en la tabla 1, durante el periodo de estudio no se presentaron muertes maternas asociadas a este tipo de evento.

Tabla 1. Características demográficas de las pacientes que presentaron Hemorragia posparto primaria en un hospital de tercer nivel de Montería 2016

\begin{tabular}{|l|c|}
\hline \multicolumn{1}{|c|}{ Variable } & $\mathrm{N}=24(100 \%)$ \\
\hline Edad & $13-42$ AÑOS \\
\hline \multicolumn{2}{|c|}{ Estado civil } \\
\hline Solteras & $11(46)$ \\
\hline Unión libre & $13(54)$ \\
\hline \multicolumn{2}{|c|}{$16(66)$} \\
\hline Área urbana & $8(33)$ \\
\hline Área rural & $18(75)$ \\
\hline & $6(25)$ \\
\hline Si & $8(33)$ \\
\hline No & $16(66)$ \\
\hline \multicolumn{2}{|c|}{ Vía del Parto } \\
\hline Nulípara & $20(83)$ \\
\hline Multípara & $4(17)$ \\
\hline & $18(75)$ \\
\hline Vaginal & $6(25)$ \\
\hline Cesárea & \\
\hline Identificación de la Hemorragia posparto \\
\hline I Nivel de atención & II o III Nivel de atención \\
\hline
\end{tabular}

Fuente: Historias clínicas, estudio caracterización de los desenlaces maternos de la hemorragia posparto primaria en un hospital de tercer nivel de Montería 2016.
El mayor número de casos de hemorragias pospartoprimariasepresentóenlasinstituciones de Primer Nivel de Atención en Salud, como se evidencia en la Tabla 2. El municipio de Montería, por ser la capital del departamento es el que reporta la mayor proporción de casos de HPP. Al caracterizar la población por sitio de procedencia, se puede apreciar que los municipios del bajo Sinú es donde se concentra el $80 \%$ de los casos reportados; este estudio también documenta casos de HPP de otros departamentos como Antioquia y Chocó.

Tabla 2. Georreferenciación de las pacientes con hemorragia posparto primaria en un hospital de tercer nivel de Montería 2016.

\begin{tabular}{|l|c|}
\hline \multicolumn{1}{|c|}{ Procedencia } & Número de Casos \\
\hline Buena Vista & 1 \\
\hline Canalete & 1 \\
\hline Ciénaga de Oro & 1 \\
\hline La Apartada & 3 \\
\hline Los Córdobas & 1 \\
\hline Montelíbano & 6 \\
\hline Montería & 2 \\
\hline Planeta Rica & 1 \\
\hline Puerto escondido & 3 \\
\hline Tierra Alta & 1 \\
\hline Quibdó & 1 \\
\hline San Juan de Urabá & 24 \\
\hline TOTAL & \\
\hline
\end{tabular}

Fuente: Historias clínicas, estudio caracterización de los desenlaces maternos de la hemorragia posparto primaria en un hospital de tercer nivel de Montería 2016.

Cuando se describen las causas de hemorragia posparto, como se puede observar en la figura 1 , el $20 \%$ de los casos identificados en los partos vaginales ocurrieron por atonía uterina, mientras la mayor proporción reportada 
corresponden a la retención de tejidos y traumatismos en el canal vaginal representando el $70 \%$ de los casos de HPP, en menor proporción, se encuentran los trastornos de la coagulación (5\%) y la inversión uterina (5\%).
Cuando la vía del parto es abdominal (Cesárea), como se muestra en la tabla 3, la mayor proporción de hemorragia ocurre por atonía uterina, esta causa representa el $75 \%$ de los casos reportados, el $25 \%$ restante corresponde a retención de tejidos placentarios o restos de membranas ovulares.

\section{PARTO POR CESÁREA}
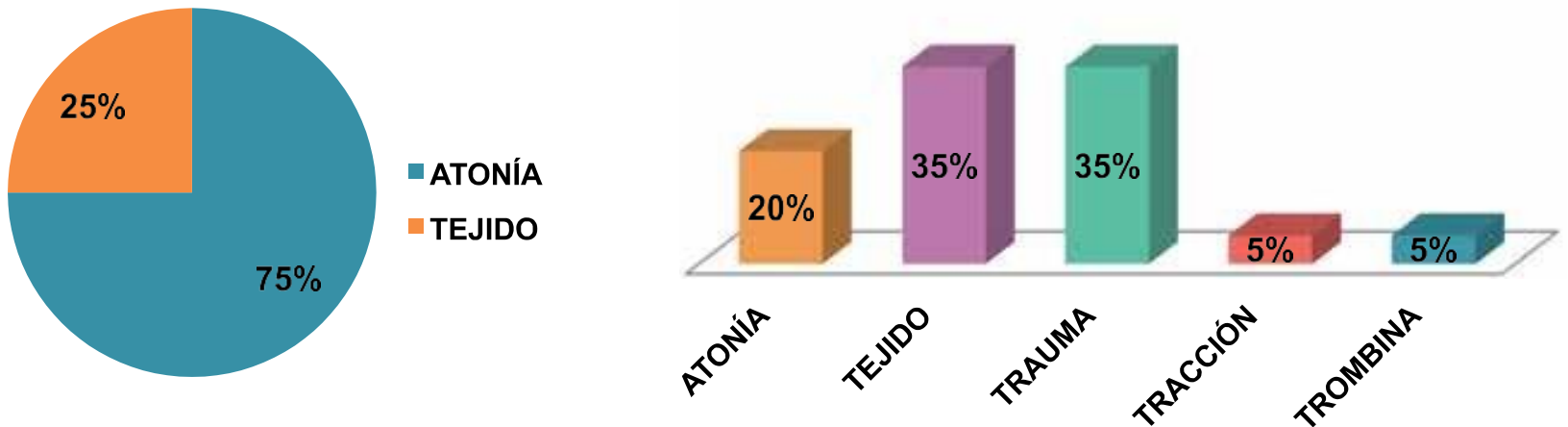

Figura 1. Distribución porcentual de las causas de hemorragia posparto primaria, según tipo de parto. Fuente: Historias clínicas, estudio caracterización de los desenlaces maternos de la hemorragia posparto primaria en un hospital de tercer nivel de Montería 2016.

Tabla 3. Causas de hemorragia posparto primaria en un hospital de tercer nivel de Montería 2016.

\begin{tabular}{|l|c|c|c|}
\hline \multirow{2}{*}{ Etiología de la HPP } & \multicolumn{2}{c|}{ Vía del parto } & \multirow{2}{*}{ Total $\mathrm{n}=24$} \\
\cline { 2 - 3 } & $\begin{array}{c}\text { Vaginal } \\
\mathrm{n}=20\end{array}$ & $\begin{array}{c}\text { Cesárea } \\
\mathrm{n}=4\end{array}$ & \\
\hline Atonía uterina & $4(20 \%)$ & $3(75 \%)$ & $7(29.1 \%)$ \\
\hline $\begin{array}{l}\text { Retención de tejidos placentarios o restos de membranas } \\
\text { ovulares }\end{array}$ & $7(35 \%)$ & $1(25 \%)$ & $8(33.3 \%)$ \\
\hline Trauma & $7(35 \%)$ & 0 & $7(29.1 \%)$ \\
\hline Tracción (Inversión uterina) & $1(5 \%)$ & 0 & $1(4.1 \%)$ \\
\hline Alteración de la coagulación * & $1(5 \%)$ & 0 & $1(4.1 \%)$ \\
\hline $\begin{array}{l}\text { *Sepresentóuna inversiónuterina, manejadaconlamaniobrade Johnson, yunapacientepresentóun trastorno } \\
\text { de la coagulación no especificado. }\end{array}$ & \\
\hline
\end{tabular}


A diferencia de lo reportado en otros estudios $(16,17)$, las causas más frecuentes de HPP en los partos vaginales identificadas en esta investigación, estén relacionadas con traumatismos en el canal vaginal (35\%) y con la retención de tejido placentario y restos de membranas ovulares (35\%). Esta situación es realmente preocupante ya que cuestiona un poco el manejo que se está realizando en la atención del parto, especialmente con la recomendación de la OMS frente el manejo activo del tercer periodo del parto.

La evidencia científica disponible sugiere que la oxitocina de 5 - $10 \mathrm{UI}$ por inyección intramuscular es el régimen de elección para la profilaxis en la tercera etapa del parto. La oxitocina intramuscular se debe administrar posterior a la salida del hombro anterior, o inmediatamente después del nacimiento del bebé y antes de realizar el pinzamiento del cordón umbilical y que éste sea cortado (18).

Por otro lado, el alumbramiento activo se ha convertido en la piedra angular para la prevención de la HPP. Esta intervención se conoce como "conducta activa del tercer estadio del parto" que consiste en realizar las siguientes medidas preventivas: La administración de un agente uterotónico profiláctico después del nacimiento de un neonato, el pinzamiento y corte temprano del cordón y la tracción controlada del cordón umbilical (18).

El masaje uterino también se incluye como parte de la conducta activa en el alumbramiento. A diferencia de la conducta activa, la conducta expectante implica esperar la aparición de signos de separación de la placenta y permite que la placenta sea expulsada en forma espontánea o con la ayuda de la estimulación de los pezones o la gravedad. En comparación con la conducta expectante, la conducta activa en el alumbramiento está asociada con una reducción considerable en la frecuencia de HPP (18). Por lo general, se supone que mediante la prevención y el tratamiento de la HPP, la mayoría de las muertes asociadas con la HPP podrían evitarse.

La estimación clínica del sangrado posparto tiene una baja sensibilidad y especificidad que conllevan el riesgo de un diagnóstico tardío (18); por esta razón, la hemorragia posparto se identifica clínicamente por la presencia de signos de choque durante el puerperio inmediato, empleando criterios modificados por Vélez et al (19), de la clasificación de Baskett para choque hipovolémico (20).

Una vez se identifica que una mujer presenta un sangrado anormal o hemorragia posparto con signos de choque, la conducta inmediata está centrada en cuatro pilares fundamentales: comunicación, reanimación, investigación de la causa y control de la hemorragia $(21,22)$. Este manejo integrado y simultaneo requiere el desarrollo de habilidades y destrezas desde el punto de vista técnico y científico por parte de los prestadores de salud que atienden la emergencia, así como el desarrollo e implementación de habilidades que faciliten y promuevanel trabajo en equipo, de manera que se optimice el tiempo de respuesta, el uso de los recursos e insumos utilizados para el manejo de la emergencia y la ejecución de las tareas (22).

La respuesta de las mujeres con HPP al tratamiento instaurado y los desenlaces maternos fue evaluada en relación con el grado de choque definido por el protocolo de código rojo obstétrico adoptado por la institución para el manejo de esta emergencia obstétrica (23). En la tabla 4, se muestra que las mujeres con mayor grado de choque, requirieron manejo avanzado y respondieron al manejo quirúrgico instaurado, el $50 \%$ de las pacientes fueron transfundidas, uno de los casosidentificados, logró demostrar la efectividad clínica del taponamiento uterino con condón y otro la efectividad clínica de la maniobra de Johnson, maniobra que consiste en tomar el fondo 
uterino invertidoyempujarlo a través del anillo cervical para restaurar su posición normal.

La presente investigación presenta limitaciones por tratarse de un estudio exploratorio basado en la consulta de registros clínicos, además de la baja incidencia del evento, la ausencia de métodos estandarizados para la medición del sangrado, los registros clínicos presentaron variabilidad en los criterios utilizados por parte del personal tratante para la identificación y determinación de los tratamientos, aun contando con un protocolo institucional para el manejo de este tipo de emergencia obstétrica.

Tabla 4. Respuesta clínica según grado de choque al momento del diagnóstico de la hemorragia posparto primaria en un hospital de tercer nivel de Montería 2016

\begin{tabular}{|c|c|c|c|c|}
\hline Grado de Choque & $\begin{array}{l}\text { Repuesta al manejo } \\
\text { medico } n(\%)\end{array}$ & $\begin{array}{l}\text { Necesidad de manejo } \\
\text { avanzado } \mathrm{n}(\%)\end{array}$ & $\begin{array}{l}\text { Necesidad de manejo } \\
\text { quirúrgico } \mathrm{n}(\%)\end{array}$ & $\begin{array}{c}\text { Total } n=24 \\
(100 \%)\end{array}$ \\
\hline $\begin{array}{c}\text { Compensado } \\
\text { Frecuencia cardiaca } \\
\text { 60-90 lat } / \text { min, Presión arterial } \\
\text { sistólica }>90 \mathrm{mmHg}\end{array}$ & $6(25)$ & $4(16.6)$ & $1^{*}(4.16)$ & $11(45.83)$ \\
\hline $\begin{array}{c}\text { Moderado } \\
\text { Frecuencia cardiaca } \\
91-100 \text { lat } / \mathrm{min}, \text { Presión arterial } \\
\text { sistólica } 80-90 \mathrm{mmHg}\end{array}$ & $0(0)$ & $4(16.6)$ & $1^{*}(4.16)$ & $5(20.83)$ \\
\hline $\begin{array}{c}\text { Leve } \\
\text { Frecuencia cardiaca } \\
\text { 101-120 lat/min, Presión } \\
\text { arterial sistólica } 70-79 \mathrm{mmHg}\end{array}$ & $0(0)$ & $1(4.16)$ & $1^{* *}(4.16)$ & $2(8.33)$ \\
\hline $\begin{array}{l}\text { Severo } \\
\text { Frecuenciacardiaca > } 120 \text { lat/ } \\
\text { min, Presión arterial sistólica } \\
70 \mathrm{mmHg}\end{array}$ & $0(0)$ & $5(20.8)$ & $1^{* * *}(4.16)$ & $6(25)$ \\
\hline
\end{tabular}

Fuente: Historias clínicas, estudio caracterización de los desenlaces maternos de la hemorragia posparto primaria en un hospital de tercer nivel de Montería 2016.

Del total de partos vaginales identificados con hemorragia posparto se observó que 20/24, pacientes se les realizó episiotomía, 7/24 mujeres presentaron desgarros perineales. Ninguna paciente tenía antecedente de HPP y solo una tuvo un neonato que pesó 4000 gramos.

La totalidad de pacientes recibieron infusión de oxitocina a una dosis inicial entre 20 y 60 $\mathrm{UI}$ (mediana de $40 \mathrm{UI}$ ), metilergonovina a una dosis mediana de $0.2 \mathrm{mg}$ misoprostol a una dosis mediana de 1000 microgramos por vía rectal. 12 pacientes (50\% de los casos) requirieron transfusión sanguínea de glóbulos rojos $\mathrm{O} \mathrm{Rh}$ - , 4 pacientes ( $17 \%$ de los casos) requirieron de ingreso a una $\mathrm{UCl}$ y el $33 \%$ de los casos restantes necesitaron manejo quirúrgico, de los cuales dos pacientes requirieron de histerectomía subtotal, ambas primigestantes adolescentes, para mayor ilustración, remítase a la tabla 5. 
Tabla 5. Desenlaces maternos de la hemorragia posparto primaria, según la vía del parto.

\begin{tabular}{|c|c|c|c|c|}
\hline Vía del Parto & Necesidad de transfusión & Manejo QCO & Ingreso a UCI & Total \\
\hline Vaginal & 10 & $\begin{array}{c}\text { 2 Histerectomía Subtotal } \\
4 \text { Legrado Uterino } \\
1 \text { Corrección De Desgarro }\end{array}$ & 3 & $20(83 \%)$ \\
\hline Cesárea & 2 & 1 Histectomía Subtutal & 1 & $4(17 \%)$ \\
\hline
\end{tabular}

Fuente: Historias clínicas, estudio caracterización de los desenlaces maternos de la hemorragia posparto primaria en un hospital de tercer nivel de Montería 2016.

El $60 \%$ de los casos de Hemorragia posparto fue identificado por el personal de enfermería, la activación de la respuesta inmediata a la hemorragia estuvo precedida por la estimación visual de las perdidas sanguíneas, las cuales fueron valoradas de manera subjetiva, el manejo convencional de la emergencia obstétrica se resolvió con la reposición del volumen sanguíneo traslaadministración decristaloides isotónicos, la corrección de las causas del sangrado y el uso de agentes uterotónicos.

\section{DISCUSIÓN}

Desde la época de Florence Nightingale la labor de la enfermera en el ambiente hospitalario ha sido imprescindible, más aún en la implementación de los códigos de respuesta inmediata (24). La enfermera juega un papel fundamentalenelequipoderespuestainmediata a la emergencia obstétrica por Hemorragia posparto en las unidades de obstetricia (25), la enfermera posee la suficiente habilidad para reconocer tempranamente los signos de choque hipovolémico en una paciente obstétrica, sin embargo se ha cuestionado mucho el juicio clínico del personal de enfermería en la estimación visual del sangrado. El personal de salud, en especial el profesional de enfermería debe ser consciente que la estimación visual de la pérdida sanguínea periparto es inexacta y que los signos y síntomas clínicos deben incluirse en la evaluación de la HPP (26).

Durante el posparto inmediato la enfermera asiste el cuidado de la mujer a través de la vigilancia estricta de los signos vitales, la valoración de la involución uterina, el fomentoa la lactancia y la vigilancia del sangrado vaginal, estas actividades se encuentran protocolizas en el cuidado de la mujer en el periodo de posparto y es una estrategia de suma importancia para el reconocimiento temprano de los signos y síntomas de choque Hipovolémico y la prevención de la hemorragia posparto.

La investigación realizada por Álvarez Franco 2013 (27), de tipo cualitativo fenomenológico interpretativo, describió la manera como las mujeres, que presentaron hemorragia postparto, perciben el cuidado prestado por el personal de enfermería durante el trabajo de parto, parto y postparto. En esta investigación, las mujeres reconocen y valoran el cuidado que ofrecen las enfermeras durante el trabajo de parto, partoy postparto, especialmente cuando viven la experiencia de presentar un episodio de hemorragia posparto porque están pendientes no solo del bienestar físico y emocional de ellas, sino también del bebé apoyándolas en su rol maternal. En esta investigación también se identificó la reciprocidad que hay entre el 
comportamiento de las mujeres y el personal de enfermería cuando se ofrece un cuidado humanizado.

El uso de agentes uterotónicos desempeña una función central en el tratamiento de la HPP. Se recomienda el masaje uterino para el tratamiento de la HPP tan pronto se diagnostique, y se recomienda la reanimación inicial con líquidos cristaloides tibios e isotónicos (28). Dentro de las habilidades técnicas, el personal de salud debe conocer el tratamiento médico adecuado e iniciarlo rápidamente con el propósito de controlar la hemorragia, restaurar o mantener una adecuada perfusión tisular y disminuir el riesgo de complicaciones (28). Teniendo en cuenta que la mayoría de los casos de hemorragia posparto se deben a dificultades con recuperar el tono uterino (hipotonía Uterina), el manejo inicial incluye además el empleo de medicamentos uterotónicos, la disponibilidad de una red de atención que permita el traslado oportuno de la mujer a un nivel de mayor complejidad para un manejo avanzado (28). En este estudio, el $75 \%$ de los casos de hemorragia posparto primaria fueron identificados en los primeros niveles de atención en Salud de la ciudad de Montería y los municipios aledaños, el traslado de estas pacientes a un nivel de atención de mayor complejidad estuvo justificado por el grado de choque y la necesidad de un manejo avanzado de las condiciones clínicas de los casos reportados.

En el departamento de Córdoba, especialmente en la ciudad de Montería el parto de bajo riesgo es atendido en el I Nivel de atención en salud, en este estudio se puede evidenciar que la mayor proporción de casos de hemorragia posparto primaria se debió a la retención de tejidos $35 \%$ y a traumatismos en el canal vaginal $35 \%$, este tipo de complicaciones está asociada con la técnica de atención delparto y con la habilidad instrumental de grupo de profesionales que realiza este procedimiento.
Cuando el diagnóstico de la hemorragia posparto corresponde a retención de tejidos placentarios o membranas ovulares, se debe efectuar lo más pronto posible la revisión y extracción de los tejidos, especialmente cuando no se tiene la precaución de asistir expulsión placentaria. En estos casos la paciente requiere anestesia general (28). Ahora bien, cuando existen traumatismo genital y desgarros ligado a un sangrado persistente, los genitales deben ser evaluados cuidadosamente y reparados, situación que requiere en muchas ocasiones de un manejo avanzado cuando no se cuenta con la suficiente experiencia por parte del personal de salud.

Con relación al manejo de la emergencia obstétrica por hemorragia posparto existe un número reducido de experiencias investigativas $(29,30,31)$, que si bien son pocas hacen una llamado a fortalecer las habilidades clínicas para la respuesta organizada de una emergencia obstétrica como lo es la Hemorragia posparto primaria. Un estudio descriptivo realizado por Mantilla 2015 (32). con el propósito de determinar del nivel de conocimiento que tenían los profesionales de enfermería de sala departosenelestándaroperacional delmanejo del código rojo, este estudio se llevó a cabo con 36 enfermeras de dos instituciones de 3 nivel de complejidad de Bogotá D.C, a las cuales se les aplicóuncuestionariode25preguntasconopción única de respuesta, el estudio concluye que el nivel deconocimiento reportadoporel grupo de estudio fue medio-bajo independientemente de los años de experiencia, el nivel de formación y el tiempo de estancia en el servicio de sala de partos, otros de los hallazgos reportados fue la necesidad de fortalecer los conceptos generales sobre hemorragia obstétrica, los medicamentos utilizados en atención de la emergencia obstétrica, el uso de equipos, la habilidad para realizar procedimientos ante una hemorragia posparto y el fortalecimiento del rol de enfermería en atención de la hemorragia posparto. 
García et al 2010 (33), reportan que el 68,9\% $(40 / 58)$ respondió al momento de activación de código rojo, sólo el 10,34\% (6/58) desconoce quiénes deben responder anteuncódigorojo, $\mathrm{El}$ $75,8 \%(44 / 58)$ sabe el volumen de cristaloides quesedebeadministrarinicialmente. El $34,48 \%$ $(20 / 58)$ desconoce las medidas que se deben implementar ante un código rojo. El $56,9 \%$ $(33 / 58)$ desconoce las medidas farmacológicas implicadas en el tratamiento del código rojo. El $60,34 \%(35 / 58)$ conoce los procedimientos quirúrgicos que se deben seguir. Sólo el $22 \%$ $(13 / 58)$ reconoce la conducta correcta en la administración de componentes sanguíneos.

En este estudio, no se evaluó la respuesta organizada del equipo de trabajo ante la hemorragia posparto, la reconstrucción de los hechos fueron recreados con el seguimiento de los registros clínicos sistematizados, y la descripción de las intervenciones que se utilizaron para el manejo de esta complicación obstétrica consignados en la historia clínica.

El manejo de la hemorragia obstétrica amerita un procedimiento sistemático, en equipo, rápido y oportuno para que en el mediano plazo se logre disminuir la morbilidad y mortalidad materna, tal como lo sostienen autores como Gosman et al 2008 (34).

La oportunidad y la calidad de la atención en el manejo de esta situación son fundamentales paradisminuir lamorbilidad maternaExtremay evitar una mortalidad materna. Para prevenirla mortalidad materna por hemorragia posparto se requiere que el equipo de respuesta inmediata (ERI), este equipo debe estar conformado por un grupo multidisciplinario, capacitado para el reconocimiento de una urgencia obstétrica, el trabajo en equipo y el uso de distintas maniobras para disminuir el riesgo de muerte materna, estando familiarizado con la atención de la urgencia obstétrica.

Martínez Rodríguez et al 2014 (35), realizaron un estudio retrospectivo/prospectivo, donde analizaron 59 casos con hemorragia obstétrica y su atención por medio de un equipo de Respuesta Inmediata (ERI). En el desarrollo de este estudio se captaron 59 pacientes con diagnóstico de hemorragia obstétrica. La media delaedaddelaspacientesfue 30 años. Elmotivo principal que originó la hemorragia obstétrica fue el desprendimiento prematuro de placenta normo inserta seguido por la atonía uterina. $\mathrm{El}$ sitio donde más frecuentemente actuó el ERI fue en la sala de partos y en $93.2 \%$ de los casos fue el médico quien inició la respuesta inmediata a la urgencia Obstétrica, el $8.5 \%$ de las mujeres con Hemorragia posparto ingresó a una unidad de cuidados intensivos y $1.7 \%$ fue trasladado a un nivel de atención de mayor complejidad. De acuerdo con los resultados obtenidos en este estudio, la aplicación del ERI fue de forma correcta, integrada y uniforme.

Ruiz Fernández et al 2015 (36), realizaron un estudio de cohorte concurrente de tipo descriptivo y prospectivo dentro de un sistema de vigilancia de hemorragia posparto basado en registros. Con el fin de describir las características demográficas, causas, intervenciones realizadas y desenlaces maternos en las mujeres que presentaronhemorragiaenlas primeras 24 horas posparto o poscesárea en un hospital de mediana complejidad. En esteestudio se presentaron 35 casos de hemorragia posparto 26 en parto vaginal y 9 en cesárea. La principal causa de hemorragia posparto fue la hipotonía uterina (82\%). El código rojo Obstétrico se activó en 11 casos (31\%), estas pacientes tuvieron morbilidad asociada para este evento, en el $82.8 \%$ de los casos el cuadro resolvió solo con manejo médico. El $11.4 \%$ requirió taponamiento uterino adicional logrando el control del sangrado. El 5,6\% de las mujeres requirió manejo quirúrgico con sutura hemostática (2.8\%) o histerectomía obstétrica (2.8\%). El índice de choque no tuvo relación con los desenlaces medidos. La incidencia de hemorragia posparto reportada fue de $2.1 \%$, menor a la reportada en la literatura. El $69 \%$ 
de las mujeres reciben manejo para hemorragia sin activar el código rojo obstétrico, siendo exitoso el tratamiento médico en la mayoría de pacientes.

\section{CONCLUSIONES}

Las causas más frecuente de HPP en los partos vaginales reportados en esta investigación están relacionadas con traumatismos en el canal vaginal y con la retención de tejido placentario y restos de membranas ovulares. Esta situación es realmente preocupante ya que cuestiona el manejoquerealizael personaldesaluddurantela atención del parto, especialmente la adherencia a la recomendación de la OMS frente el manejo activo del tercer periodo del parto.

La activación de la respuesta inmediata a la hemorragia Posparto estuvo precedida por la estimación visual de las perdidas sanguíneas, las cuales fueron valoradas de manera subjetiva, para este estudio el manejo de la emergencia obstétrica se resolvió con la reposición del volumen sanguíneo tras la administración de cristaloides isotónicos, la corrección de las causas del sangrado, la revisión y extracción de restos placentariosyovulares bajoanestesia general y el uso de agentes uterotónicos.

Las mujeres con mayor grado de choque, requirieron manejo avanzado y respondieron al manejo quirúrgico instaurado, el $50 \%$ de las pacientes fueron transfundidas, uno de los casos identificados, logró demostrar la efectividad clínica del taponamiento uterino con condón y otro la efectividad clínica de la maniobra de Johnson.

A pesar de existir un amplio espectro de intervenciones para la corrección de las causas de Hemorragia posparto, queda claro que si no se cuenta con unentrenamiento adecuado para llevar a cabo estas acciones, los esfuerzos instaurados serían insuficientes para evitar una mortalidad materna.
La coordinación de las acciones, el reconocimiento temprano de los signos de choque y el manejo oportuno de la hemorragia posparto son elementos cruciales para salvaguardar la vida de una mujer con hemorragia posparto, por otro lado, los equipos desalud de las unidades de serviciosobstétricos siempre deben estar preparados para responder a este tipo deemergencias.

A pesar de contar con un protocolo de atención para el manejo de la emergencia obstétrica por hemorragia posparto a nivel institucional, sigue prevaleciendo una amplia variabilidad en la forma como el personal de salud da respuesta a este tipo de complicaciones, es posible que este tipo de conductas se encuentre permeado por las escuelas de formación y la trayectoria de quienes llevanel liderazgodeestasacciones, de hecho, esta situación no es el cuello de botella en el abordaje de la emergencia obstétrica, lo es más bien , la manera como el equipo de trabajo de las unidades obstétricas se organizan para responder de manera efectiva y segura ante un caso de hemorragia posparto.

\section{RECOMENDACIONES}

Existe la necesidad de ampliar la descripción del comportamiento de la hemorragia posparto a través de estudios prospectivos, especialmente documentar como se coordinan las acciones de los equipos de respuesta inmediata en la secuencia temporal del manejo de la hemorragia obstétrica. Así mismo involucrar otras variables clínicas de interés, que permitan la predicción de los desenlaces maternos como el Índice de Choque (IC) teniendo en cuenta que la estimación visual de la pérdida sanguínea periparto es inexacta y que los signos y síntomas clínicos deben incluirse en la evaluación de la HPP.

Favorecer el entrenamiento del personal que labora en las unidades obstétricas a través de intervenciones educativas que utilicen la 
simulación clínica como estrategia para el desarrollo de habilidades y destrezas en la toma de decisiones y el manejo clínico de la hemorragia posparto.

\section{CONFLICTO DE INTERÉS}

Los autores declaran no tener conflicto de interés.

\section{REFERENCIAS}

1. American College of Obstetricians and Gynecologists. ACOG Practice Bulletin: Clinical Management Guidelines for Obstetrician-Gynecologists Number 76, October 2006: postpartum hemorrhage. Obstet Gynecol. 2006 Oct; 108 (4):1039-47.

2. Say L, Chou D, Gemmill A, Tunçalp Ö, Moller A-B, Daniels J, et al. Global causes of maternal death: a WHO systematic analysis. Lancet Glob Health. 2014 Jun; 2(6): 323-333.

3. Carroli G, Cuesta C, Abalos E, Gulmezoglu AM. Epidemiology of postpartum haemorrhage: a systematic review. Best Pract Res Clin Obstet Gynaecol. 2008 Dec; 22(6):999-1012.

4. Karrie E. Francois. Obstetrics: Normal and Problem Pregnancies. Seventh. Vol. Chapter 18. 395-424 p.

5. WHO Recommendations for the Prevention and Treatment of Postpartum Haemorrhage [Internet]. Geneva: World Health Organization; 2012 [cited 2016 Dec 15]. (WHO Guidelines Approved by the Guidelines Review Committee). Available from: http: //www.ncbi.nlm.nih. gov/books/NBK131942/.

6. Gosman GG, Baldisseri $M R$, Stein $\mathrm{KL}$, Nelson TA, Pedaline SH, Waters JH, et al Introduction of an ob- stetric-specific medical emergency team for obstetric crises: implementation and experience. Am J Obstet Gynecol 2008; 198:367.e1-367.e7.

7. Postpartum Haemorrhage, Prevention and Management (Green-top Guideline No.52) [Internet]. Royal College of Obstetricians \&amp; Gynecologists. [Cited 2016 Dec 15]. Available from: https://www.rcog. org.uk/en/guidelines-research-services/ guidelines/gtg52/

8. American College of Obstetricians and Gynecologists. ACOG Practice Bulletin: Clinical Management Guidelines for Obstetrician-Gynecologists Number 76, October 2006: postpartum hemorrhage. Obstet Gynecol. 2006 Oct; 108 (4):1039-47.

9. Revista colombiana de obstetricia y ginecología. Guía de práctica clínica para la prevención yel manejo de la hemorragia posparto y complicaciones del choque hemorrágico. Scielo. Vol.64, no.4, 2013. Consultado en: http: / / www.scielo.org.co/ scielo.php?script=sci_arttext\&pid=S003474342013000400004

10. Secretaria distrital de salud. Guía de hemorragia postparto Código Rojo. 2013 - 2014. Consultado en: http://www. saludcapital.gov.co/DDS/Publicaciones/ Guia\%20Maternidad-Codigo\%20 Rojo_7A.pdf

11. Tratamiento para la hemorragia postparto primaria (Revisión Cochrane traducida). Cochrane Database of Systematic Reviews 2014 Issue 2. Art. No.: CD003249. DOI: $10.1002 / 14651858 . C D 003249$.

12. Postpartum Hemorrhage, Prevention and Management (Green-top Guideline No.52) [Internet]. Royal College of Obstetricians \& amp; Gynecologists. [cited 2017 Jun 15]. Available from: https://www.rcog. org.uk/en/guidelines-research-services/ guidelines/gtg52/ 
13. Organización Mundial de la Salud (OMS). Tratamiento para hemorragia postparto primaria. 2015. Consultado: 16 /05/16. Consultado en: http://apps.who. int/rhl/pregnancy_childbirth/childbirth/ postpartum_hae morrhage/sfcom/es/

14. Vélez, GA, Agudelo B, Gómez, JG, Zuleta, J. Código rojo: guía para el manejo de la hemorragia obstétrica. Rev Colomb Obstet Ginecol. 2016 Feb 16; 60(1):34-48.

15. InstitutoNacional deSalud2014. Protocolo de vigilancia en salud pública morbilidad materna extrema. Disponible EN: www. ins.gov.co/...vigilancia/.../Protocolos $\% 20$ SIVIGILA/PRO\%20Morbilidad\%20Ma

16. Carroli G, Cuesta C, Abalos E, Gulmezoglu AM. Epidemiology of postpartum haemorrhage: a systematic review. Best Pract Res Clin Obstet Gynaecol. 2008 Dec; 22(6):999-1012.

17. Ruiz $F$ et al 2015. Evaluación de los Desenlaces Maternos y Efectividad del Manejo de la Hemorragia Posparto en un Hospital de Segundo Nivel de Bogotá. Repositorio biblioteca Universidad Nacional de Colombia

18. Mavrides E, Allard S, Chandraharan E, Collins P, Green L, Hunt BJ, Riris S, Thomson AJ on behalf of the Royal College of Physicians. Prevention and management of postpartum haemorrhage. BJOG 2016; DOI: .10.1111/ 1471-0528.14178.

19. Vélez, GA, Agudelo, B, Gómez, JG, Zuleta, JJ. Código rojo: guía para el manejo de la hemorragia obstétrica. Rev Colomb Obstet Ginecol. 2016 Feb 16; 60(1):34-48.

20. Baskett PJ. $A B C$ of major trauma. Management of hypovolaemic shock. BMJ 1990; 300:1453-7.
21. Rubio, JA, Gaitán, HG, Rodríguez, N. Concordancia entre la estimación visual y la medición del volumen recolectado en una bolsa del sangrado intraparto en mujeres con parto normal en Bogotá, Colombia, 2006. Rev Colomb Obstet Ginecol. 2016 Jul 5; 59(2):92-102.

22. Le Bas A, Chandraharan E, Addei A, Arulkumaran S. Use of the "obstetric shock index" as an adjunct in identifying significant blood loss in patients with massive postpartum hemorrhage. Int J Gynaecol Obstet Off Organ Int Fed Gynaecol Obstet. 2014 Mar;124(3):253-5.

23. Ministerio de protección social 2013. Guía de práctica clínica para la prevención, detección temprana y tratamiento de las complicaciones del embarazo, parto y puerperio. Disponible EN: https:// www. minsalud.gov.co/sites/rid/.../Guía. completa.Embarazo.Parto.2013.pdf

24. Boyd, J. Florence Nightingale's. Remarkable life and work. The Lancet. 2008; 372:1375-6.

25. Carlson, K. Knowledge assessment and preparation for the certiied emergency nurses examination. J Emerg Nurs. 2010; 36:38-9.

26. Rubio, JA, Gaitán, HG, Rodríguez, N. Concordancia entre la estimación visual y la medición del volumen recolectado en una bolsa del sangrado intraparto en mujeres con parto normal en Bogotá, Colombia, 2006. Rev Colomb Obstet Ginecol. 2016 Jul 5; 59(2):92-102.

27. Álvarez, C. Cómo describen el cuidado de enfermería las mujeres que presentaron hemorragia postparto. 2013. Consultado en: http://aquichan.unisabana.edu.co/ index.php/aquichan/article/view/229 
28. Postpartum Haemorrhage, Prevention and Management (Green-top Guideline No.52) [Internet]. Royal College of Obstetricians \&amp; Gynecologists. [Cited 2016 Dec 15]. Available from: https://www.rcog. org.uk/en/guidelines-research-services/ guidelines/gtg52/

29. Navarro, Castillo. Código rojo, un ejemplo de sistema de respuesta rápida. Rev. Col. Anest. Febrero -abril 2010. Vol. 38 - No. 1: 86-99.

30. Ruiz, RA, Cruz, PR, Aguilar, L, Veloz, MG. Mortalidad materna. En: Tena, G, ed. Editoral Alfil SA de CV: México. 2013; pp 89-111.

31. Gosman GG, Baldisseri $M R$, Stein $\mathrm{KL}$, Nelson TA, Pedaline SH, Waters JH, et al Introduction of an ob- stetric-specific medical emergency team for obstetric crises: implementation and experience. Am J Obstet Gynecol 2008; 198:367.e1367.e7

32. Martínez, Rodríguez et al. Equipo de respuesta inmediata, análisis de 59 casos con hemorragia obstétrica. Rev Med Inst Méx Seguro Soc. 2015; 53(2):132-5

33. García A, Navarro R, Eslava, J. Encuesta sobre código rojo en cinco instituciones de salud de Bogotá. 2010. Consultado En: www.sciencedirect.com/science/article/ pii/S0120334710810044

34. Gosman GG, Baldisseri MR, Stein $\mathrm{KL}$, Nelson TA, Pedaline SH, Waters JH, et al Introduction of an ob- stetric-specific medical emergency team for obstetric crises: implementation and experience. Am J Obstet Gynecol 2008; 198:367.e1367.e7.

35. Martínez Rodríguez et al. Equipo de respuesta inmediata, análisis de 59 casos con hemorragia obstétrica. Rev Med Inst Méx Seguro Soc. 2015; 53(2):132-5

36. Ruiz Fernández et al 2015. Evaluación de los Desenlaces Maternos y Efectividad del Manejo de la Hemorragia Posparto en un Hospital de Segundo Nivel de Bogotá. Repositorio biblioteca Universidad Nacional de Colombia. 